\title{
A Hybrid Approach for Scattering of Elastic Waves by Three-Dimensional Irregularities of Arbitrary Shape
}

\author{
Tomi K. Mossessian* and Marijan Dravinski \\ Department of Mechanical Engineering, University of Southern California, \\ Los Angeles, California, U.S.A.
}

\begin{abstract}
A hybrid technique is developed for studying scattering of elastic waves by non-axisymmetric three-dimensional near-surface inhomogeneities. The technique combines an indirect boundary integral equation method with the finite element approach. Special emphasis is placed on inhomogeneities in the form of dipping layers embedded in a half-space and subjected to plane incident $\mathrm{P}, \mathrm{SV}, \mathrm{SH}$, and Rayleigh waves.

The accuracy and efficiency of the hybrid technique are examined through several numerical examples. The comparisons with the results obtained by a boundary integral equation method validate the accuracy of the hybrid technique. The versatility of the method is demonstrated by considering several types of inhomogeneous basins containing multiple horizontal and dipping layers. It is found that the numerical efficiency of the hybrid technique becomes much higher than that of the boundary integral equation methods as the structure of the inhomogeneities gets more complex.
\end{abstract}

\section{Introduction}

The extent of the damage in the central part of Mexico City due to the Michoacan, Mexico earthquake of September 19, 1985, was remarkably severe considering the long epicentral distance of $350 \mathrm{~km}$ (Anderson et al., 1986). Nearly all the buildings that collapsed during the earthquake of September 19, were located in the central portion of Mexico City, while the damage in the surrounding area was minimal. The central part of Mexico City is founded on a lake bed zone formed by clay deposits which are considerably softer than the alluvium of the surrounding hilly zone. In the two second period, the acceleration response at the lake zone was about ten times greater than that of outlying districts (Anderson et al., 1986). Thus, it appears that subsoil structure played an important role in the resulting pattern and extent of damages in Mexico City during the earthquake of September 19, 1985.

Observations from previous earthquakes in the Valley of Mexico (Zeevaert, 1964) show that in spite of differences in source mechanism and epicentral distances, accelerograms at the lake bed zone are similar with the low frequency oscillations known as resonant excitation of the sedimentary basin (Anderson et al., 1986). Analysis of

Received November 20, 1990; Accepted June 30, 1992

* To whom correspondence should be addressed. Presently at Structural Research and Analysis Corp., 1661 Lincoln Blvd., Suite 200, Santa Monica, CA 90404, U.S.A. 
earthquake damages at other locations, e.g., Lima, Peru earthquake of 1974 (Repetto et al. 1980), indicates that the areas of intense damage atop alluvium can be highly localized. Since many other highly populated areas are located on sedimentary basins, the study of their response to seismic waves is of great interest in earthquake engineering and strong ground motion seismology (Aki, 1988).

Experimental studies of Kagami et al. (1982), Kagami et al. (1986), and King and Tucker (1984), as well as theoretical studies by Bouchon and Aki (1977), Bard and Bouchon (1980a, b) and Dravinski (1982a, b, c, 1983) have shown that presence of sediment-filled valley may cause very large localized amplification of surface ground motion. Studies of microtremors and strong ground motion in deep alluvial basins by Kagami et al. (1982), Kagami et al. (1986), King and Tucker (1984), and Bard and Bouchon (1985) show that there appears to be a resonant-type behavior of the valley. This can be explained only if there are interactions between horizontal and vertical waves within the valley. Thus, for thorough understanding of seismic response of deep alluvial valleys it is necessary to study the problem of amplification of the surface ground motion by subsurface irregularities using two- and three-dimensional models.

Modeling the amplification of seismic waves due to scattering by subsurface irregularities is characterized by considerable complexities. Better understanding of the site amplification effects requires more realistic models, which in turn should account precisely for complex geometry and the material properties of the actual alluvial valley. For that reason, it is necessary to develop methods capable of incorporating these requirements in studying the problems of site amplification effects.

Scattering of seismic waves by an alluvial valley may be solved analytically or numerically. Analytical solutions are limited to very simple geometries and linear materials (e.g., Trifunac, 1971). Most commonly used numerical methods, finite elements and finite differences, on the other hand, require the discretization of the entire solution domain. This makes such procedures inefficient for geotechnical problems which involve very large characteristic length. However, they have the advantage in their applicability to problems with complex geometries and varying material properties (e.g., Smith, 1975; Zahradnik and Hron, 1987).

For the problems of scattering of seismic waves by irregularities with moderately shallow slopes Aki and Larner (1970) have developed a technique using the so-called Rayleigh hypothesis. The method is applicable to wavelengths which are either larger than or of the order of the dimension of the irregularities. Bouchon (1973) and Bard and Bouchon $(1980 \mathrm{a}, \mathrm{b})$ used this method to study the seismic response of sediment-filled valleys subjected to incident $\mathrm{P}, \mathrm{SV}$, and $\mathrm{SH}$ waves. Bouchon and Aki (1977) extended the Aki-Larner method to study the near field of a seismic source in a layered medium with irregular interfaces. Bard and Gariel (1986) have developed an extension of the technique to study seismic response of basins with large vertical velocity gradient. They presented results for SH waves only.

Other methods shown to be very effective for studying the wave scattering in geotechnical problems involving unbounded domains are the boundary integral equation methods (BIEM) (Dravinski, $1982 \mathrm{a}, \mathrm{b}, \mathrm{c}, 1983$; Dravinski and Mossessian, 1987 a, b; Rizzo et al., 1985; Sánchez-Sesma and Rosenblueth, 1979; Wong, 1982). These methods require only discretization of the boundary of the scatterers (Cole et al., 1978), 
and the rediation conditions at infinity can be modeled exactly. A disadvantage of BIEM is the large computational efforts needed for evaluation of the half-space Green's functions. For plain strain and three-dimensional models the Green's functions cannot be expressed in a simple form. Several alternative techniques have been proposed to avoid this difficulty. One way is to use the full-space Green's functions and then impose the traction-free boundary conditions on part of the free surface (Niwa et al., 1986). This approach has several drawbacks. First, the required size of the free surface to stimulate a half-space is not clear beforehand since it is dependent upon frequency, the shape of the irregularity and the type of incident wave. Therefore, extensive testing is required for each case. Another disadvantage of this approach becomes apparent in the three-dimensional cases (Niwa and Hirose, 1987). Since the required number of boundary elements on the free surface becomes very large (larger than what is required for the scatterer itself), the size of the Green's function matrix needed for this method grows substantially. Therefore, required amount of memory and computation time is greatly increased. Kawase and Aki (1989) have used an alternate approach to study transient responses of two-dimensional sedimentary basin. The technique combines the direct boundary element method with the discrete wavenumber Green's functions. In this approach the infinite integrals are transformed into infinite sums over discrete wavenumbers under the assumption of periodicity (Bouchon and Aki, 1977). Due to periodicity assumption, the solution is valid until the arrival time of scattered waves from adjacent irregularities. Therefore, detailed testing is needed to determine a suitable periodicity length which avoids contamination from neighboring fictitious structures.

Another boundary method suitable for problems of scattering of elastic waves in an infinite media is the wave function expansion approach (Eshraghi and Dravinski, 1988 a, b; Sánchez-Sesma, 1983). The method makes use of the C-complete family of wave functions (Herrera and Sabina, 1978) which can be evaluated very efficiently. However, the wave functions in general do not satisfy the traction-free boundary conditions. These boundary conditions have to be imposed on part of the free surface. Therefore, the disadvantages of this method are similar to the case of the BIEM with use of full-space Green's functions mentioned earlier.

For high frequency range, the ray methods and Gaussian beam technique appear to be very effective (e.g., Hong and Helmberger, 1978; Moczo et al., 1987; Nowack and Aki, 1984). These methods are relatively fast and they are applicable to inhomogeneous basins with complex geometries. As demonstrated by Moczo et al. (1987) such techniques cannot produce correct results for wavelengths larger than the minimum radius of curvature of the basin boundary. However, in higher frequency range these methods can compliment other low frequency methods mentioned earlier (BIEM, discrete wavenumber method, finite element and finite differences).

In recent years, hybrid techniques which combine the boundary integral equation methods (BIEM) with the finite element approach have proved to be very effective for studying various problems of interest in engineering mechanics, earthquake engineering and seismology (e.g., Beskos and Spyrakos, 1984; Kobayashi et al., 1986; Mossessian and Dravinski, 1987). The main advantage of the hybrid techniques is that it utilizes the versatility of the finite element method for detailed modeling of the near field and the effectiveness of the BIEM in the far field (Berg, 1984; Zienkiewicz et al., 1977). For

Vol. 40, No. 1, 1992 
extensive review of the literature on the subject the reader is referred to articles by Wong et al. (1985), Beskos (1987), and Mossessian and Dravinski (1987). These papers show that at the present time, there are very few studies that have applied the hybrid techniques to problems of scattering of elastic wave by non-axisymmetric threedimensional near-surface irregularities. One of the most complex models considered in the literature to date is due to Kobayashi et al. (1986), who investigated the steady state response of a non-axisymmetric three-dimensional structure embedded in a viscoelastic half-space. They applied a hybrid technique which utilizes a direct BIEM using full-space Green's functions. The advantage of this approach is that it avoids the extensive computational efforts needed for calculation of the half-space Green's functions (Dravinski and Mossessian, 1988). However, one needs to impose the traction-free boundary conditions on part of the free surface, which results in the drawbacks discussed earlier.

In this paper, applicability of a hybrid method to problems of scattering of elastic waves by non-axisymmetric three-dimensional subsurface inhomogeneities is investigated. The present work is an extension of the recent study done by Mossessian and Dravinski (1987) in which they investigated seismic responses of two-dimensional dipping layers of arbitrary shapes. The hybrid method combines an indirect boundary integral equation approach (Dravinski and Mossessian, 1987a; Mossessian, 1989; Mossessian and Dravinski, 1989) with the finite element technique. Incident plane harmonic P, SV, $\mathrm{SH}$, and Rayleigh waves are assumed. General formulation of the method is presented. Testing of the accuracy of the technique is performed through comparisons with the results obtained by the indirect boundary integral equation method (Mossessian, 1989) for a homogeneous dipping layer. The capability of the method is demonstrated by considering several types of inhomogeneous basins containing multiple horizontal and dipping layers. The effects of horizontal and dipping type layering on seismic response of the basin are examined. Finally, the efficiency of the method in comparison to the boundary methods is discussed.

\section{Statement of the Problem}

Geometry of the problem is depicted by Fig. 1(a). Interior region $D_{\mathrm{I}}$ is assumed to include all the irregularities which may contain inhomogeneous and nonisotropic materials. Material of the exterior region of the half-space $D_{\mathrm{E}}$ is assumed to be linearly elastic, homogeneous and isotropic. Boundary $\mathrm{B}$ defines the interface between regions $D_{\mathrm{I}}$ and $D_{\mathrm{E}}$. Incident wave is assumed to be a plane harmonic $\mathrm{P}, \mathrm{SV}, \mathrm{SH}$ or Rayleigh wave.

Equation of motion for a steady state wave propagation is specified by

$$
\operatorname{div} \sigma+\rho \omega^{2} u=0 ; \quad x \in D_{\mathrm{I}} \cup D_{\mathrm{E}},
$$

where $\sigma$ and $\boldsymbol{u}$ denote the stress tensor and displacement vector, respectively, $\boldsymbol{x}$ is a position vector, $\rho$ is the mass density, and $\omega$ is the circular frequency. Stress-free boundary conditions along the surface of the half-space are specified by

$$
\sigma_{x z}=\sigma_{y z}=\sigma_{z z}=0 ; \quad z=0 \text { and } x \in D_{\mathrm{I}} \cup D_{\mathrm{E}} .
$$

Usual radiation conditions should be satisfied by the scattered wave field at infinity. 


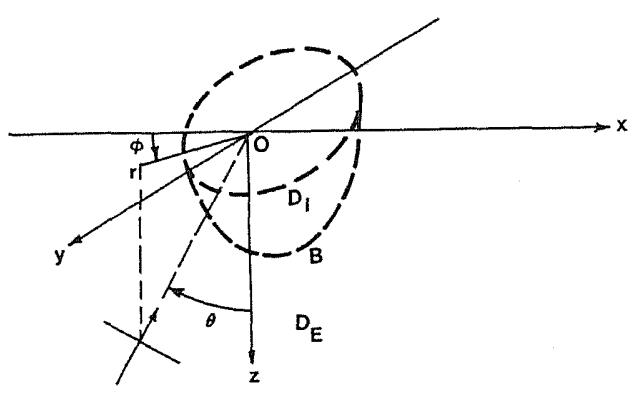

(a)

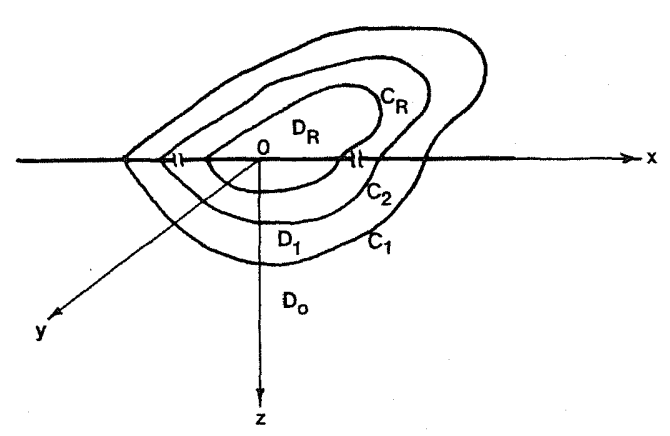

(b)

Fig. 1. (a) Geometry of the problem. (b) Basin model.

For this study, we choose region $D_{\mathrm{I}}$ to consist of several horizontal or dipping layers $\left(D_{i}, i=1, \cdots, R\right)$ which are perfectly bonded together, overlaying a half-space $D_{0}$ (Fig. 1(b)). Interfaces $C_{i}$ between the layers need not be smooth. Continuity of the displacement and the traction field along interfaces $C_{j}, j=1,2, \cdots, R$ is specified by

$$
\begin{array}{cc}
u_{j-1}^{+}=u_{j}^{-} ; & x \in C_{j} \\
t_{j-1}^{v^{+}}=t_{j}^{v^{-}} ; & x \in C_{j},
\end{array}
$$

where the subscripts + and - denote that the interface is being approached from the outside and the inside, respectively, and $t_{j}^{v}$ denotes the traction vector at a surface $C_{j}$ with an outward unit normal $v_{j}$.

\section{Method of Solution}

The interior region $D_{1}$, is modeled by using the finite element method and the exterior region $D_{\mathrm{E}}$ is modeled by using an indirect boundary integral equation approach. Complete solution of the problem is then obtained by imposing the continuity conditions along the boundary $B$.

The finite element analysis of the interior region is considered first. 


\section{Finite Element Analysis}

By discretizing the continuum of the interior region $D_{\mathrm{I}}$ into a set of finite elements, the equation of motion for each element can be written in the following form (Zienkiewicz, 1977):

$$
K_{e}^{*} u_{e}-\left(\omega^{2} M_{e} u_{e}=r_{e}^{*} ; x \in D_{1},\right.
$$

where $\boldsymbol{K}_{\boldsymbol{e}}^{*}$ and $\boldsymbol{M}_{e}$ are the stiffness and mass matrices, respectively, $\boldsymbol{r}_{e}^{*}$ is the vector of nodal point forces due to surface tractions, and $\boldsymbol{u}_{\mathrm{e}}$ contains the nodal displacements of the element.

Applying the piecewise-uniform traction approximation (i.e., the tractions are assumed to be constant along the face of each element) at the boundary of an element, $r_{e}^{*}$ reduces to

$$
r_{e}^{*}=R_{e} t_{e}^{v}
$$

where $R_{e}$ is the matrix which transforms the traction vector $t_{e}^{v}$ into the nodal force vector $r_{e}^{*}$. Hence, the equation of motion (5) can be written in the following form:

$$
K_{e} u_{e}=R_{e} t_{e}^{v},
$$

where the matrix $\boldsymbol{K}_{c}$ is defined by

$$
K_{e}=K_{e}^{*}-\omega^{2} M_{e} .
$$

The matrices $K_{e}$ and $\boldsymbol{R}_{e}$ are computed for each element and assembled into global matrices $\boldsymbol{K}$ and $\boldsymbol{R}$. Therefore, the equation of motion becomes

$$
\boldsymbol{K u}=\boldsymbol{R} \boldsymbol{t}^{v} ; \boldsymbol{x} \in D_{\mathrm{I}} .
$$

Here, $u$ and $t^{v}$ represent all the nodal displacements and tractions in the region $D_{\mathrm{I}}$, respectively. The nodal displacement $\boldsymbol{u}_{\mathrm{B}}$ at the boundary $\mathrm{B}$ can be separated from the interior nodal displacement $\boldsymbol{u}_{\mathrm{I}}$. Subsequently, the nodal displacement vector $\boldsymbol{u}$ becomes

$$
u=\left(u_{\mathrm{I}}, u_{\mathrm{B}}\right)^{\mathrm{T}},
$$

where the superscript $T$ denotes the transpose. Partitioning of the matrices in Eq. (9) results in the following equation:

$$
\left[\begin{array}{ll}
K_{\mathrm{II}} & K_{\mathrm{IB}} \\
K_{\mathrm{BI}} & K_{\mathrm{BB}}
\end{array}\right]\left[\begin{array}{l}
u_{\mathrm{I}} \\
u_{\mathrm{B}}
\end{array}\right]=\left[\begin{array}{ll}
R_{\mathrm{II}} & R_{\mathrm{IB}} \\
R_{\mathrm{BI}} & R_{\mathrm{BB}}
\end{array}\right]\left[\begin{array}{l}
t_{\mathrm{I}}^{v} \\
t_{\mathrm{B}}^{v}
\end{array}\right] .
$$

Since for the problem at hand only the boundary B would be subjected to surface tractions, there would be no nodal forces of the interior nodes, i.e., $t_{\mathrm{I}}^{\mathrm{y}}=\boldsymbol{R}_{\mathrm{IB}}=\boldsymbol{R}_{\mathrm{BI}}=0$. Thus, $u_{1}$ can be eliminated from Eq. (11) to obtain the following result:

$$
\boldsymbol{L} \boldsymbol{u}_{\mathrm{B}}=\boldsymbol{R}_{\mathrm{BB}} t_{\mathrm{B}}^{v},
$$

where

$$
L=-K_{\mathrm{BI}} K_{\mathrm{II}}^{-1} K_{\mathrm{IB}}+K_{\mathrm{BB}} .
$$


This completes the analysis of the finite element formulation for the interior region $D_{\mathrm{l}}$. It should be emphasized that Eq. (12) relates the nodal displacements $\boldsymbol{u}_{\mathrm{B}}$ with nodal tractions $t_{\mathrm{B}}^{v}$ on the boundary $\mathrm{B}$.

Formulation of the problem in the exterior region $D_{\mathrm{E}}$ is considered next.

\section{Boundary Integral Equation Analysis}

The total displacement field in the exterior region is specified by

$$
\boldsymbol{u}_{\mathrm{E}}=\boldsymbol{u}_{\mathrm{E}}^{\mathrm{ff}}+\boldsymbol{u}_{\mathrm{E}}^{\mathrm{s}} ; \quad \boldsymbol{x} \in D_{\mathrm{E}},
$$

where the superscripts ff and s denote the free and the scattered wave field, respectively. Following an indirect boundary integral equation approach (Dravinski and Mossessian, 1987a; Mossessian and Dravinski, 1989) the scattered field is assumed to be generated from distribution of unknown tractions $f(y)$ on an auxiliary surface $S_{\mathrm{a}}$ inside the surface B. Hence the scattered displacement field in the half-space can be written in the form

$$
u_{\mathrm{E}}=\int_{S_{\mathrm{a}}} G(x, y) f(y) \mathrm{d} S_{\mathrm{a}}(y) ; \quad x \in D_{\mathrm{E}},
$$

where $G$ is a half-space displacement Green's function tensor (Aki and Richards, 1980). The element $G_{i j}(x, y)$ corresponds to the $i$-th component of the displacement vector at $x$ due to a unit harmonic force at $y$ acting in the $j$-th direction. Theoretical development of these Green's functions is rather involved and their complete explicit forms can be found in the article by Mossessian and Dravinski (1989).

Choosing $f(y)$ to be concentrated at discrete points of the surface $S_{\mathrm{a}}$, it can be shown (Mossessian, 1989; Mossessian and Dravinski, 1989) that the total wave field takes the following form:

$$
u_{\mathrm{E}}=u_{\mathrm{E}}^{\mathrm{ff}}+G\left(x, x_{m}\right) f^{\mathrm{m}} ; x_{m} \in S_{\mathrm{a}}, m=1,2, \cdots, M .
$$

Here $x_{m}$ are chosen and the unknown force magnitudes $f^{\mathrm{m}}$ are yet to be determined for all $\mathrm{m}$. Throughout, summation over repeated indices $\mathrm{m}$ is understood. Using Eq. (16), the components of total traction along the surface $S_{\mathrm{I}}$ become

$$
t_{\mathrm{E}}=t_{\mathrm{E}}^{\mathrm{ff}}+T\left(x, x_{m}\right) f^{\mathrm{m}} ; \boldsymbol{x} \in \boldsymbol{B} ; m=1,2, \cdots, M,
$$

where $t^{\mathrm{ff}}$ is the free-field traction vector and $T$ is the half-space traction Green's function tensor. The component $T_{i j}\left(x, x_{m}\right)$ corresponds to the $i$-th component of the traction vector at $\boldsymbol{x}$ due to unit harmonic force at $\boldsymbol{x}_{m}$ acting in the $j$-th direction. For simplicity, superscript $v$ is suppressed. By choosing $N$ observation points on boundary $\mathrm{B}$ (to coincide with the boundary nodes of the finite element model), corresponding displacement and traction fields can be stated as follows:

$$
\begin{gathered}
u_{\mathrm{E}}=u_{\mathrm{E}}^{\mathrm{ff}}+G_{\mathrm{u}} f^{\mathrm{t}} \\
t_{\mathrm{E}}=t_{\mathrm{E}}^{\mathrm{ff}}+G_{\mathrm{t}} f^{\mathrm{t}} \\
f^{\mathrm{t}}=\left(f^{1}, f^{2}, \cdots, f^{M}\right)^{\mathrm{T}} .
\end{gathered}
$$

Here, vector $f^{t}$ (of order $3 M \times 1$ ) contains all the unknown force magnitudes. 
Matrices $G_{\mathrm{u}}$ and $G_{\mathrm{t}}$ (of order $3 N \times 3 M$ ), and vectors $\boldsymbol{t}_{\mathrm{E}}^{\mathrm{ff}}$ and $\boldsymbol{u}_{\mathrm{E}}^{\mathrm{ff}}($ of order $3 N \times 1$ ) are all known. Continuity of the displacement and traction field along the boundary $\mathrm{B}$ requires that

$$
\begin{aligned}
& u_{\mathrm{E}}=u_{\mathrm{B}} ; \quad x \in B \\
& t_{\mathrm{E}}=t_{\mathrm{B}} ; \quad x \in B,
\end{aligned}
$$

where $t_{\mathrm{E}}$ and $\boldsymbol{u}_{\mathrm{E}}$ are given by Eqs. (18) and (19). Substituting Eqs. (18) and (19) into Eq. (12), via Eqs. (20) and (21), it follows that

$$
L\left[u_{\mathrm{E}}^{\mathrm{ff}}+G_{\mathrm{u}} f^{t}\right]=R_{\mathrm{BB}}\left[t_{\mathrm{E}}^{\mathrm{ff}}+G_{\mathrm{t}} f^{\mathrm{t}}\right] .
$$

This last result can be written as

$$
G_{\mathrm{A}} f^{\mathrm{t}}=\boldsymbol{a},
$$

where

$$
\begin{aligned}
& G_{\mathrm{A}}=L G_{\mathrm{u}}-R_{\mathrm{BB}} G_{\mathrm{t}} \\
& a=R_{\mathrm{BB}} t_{\mathrm{E}}^{\mathrm{ff}}-L u_{\mathrm{E}}^{\mathrm{ff}} .
\end{aligned}
$$

By choosing $N$ greater than $M$, Eq. (23) is solved in the least square sense. Once the magnitude of forces $f^{t}$ are known, the displacement and the stress fields in region $D_{\mathrm{E}}$ can be calculated through use of Eqs. (16) and (17). The displacement field in the interior region $D_{\mathrm{I}}$ is obtained by using Eq. (11).

\section{Numerical Results and Discussion}

Shape of the linearly elastic dipping layer is chosen to be in the form of a semi-prolate with a flat lower section. This geometry can be defined in the following parametric form:

for $0<z<c$

$$
\begin{gathered}
x=a \cos (t) \cos (s) \quad y=b \cos (t) \sin (s) \quad z=b \sin (t) \\
0<s<2 \pi, \quad 0<t<t_{i}, t_{i}=\sin ^{-1}(c / b)
\end{gathered}
$$

for $z=c$

$$
x=a \cos \left(t_{i}\right) \cos (s), \quad y=b \cos \left(t_{i}\right) \sin (s),
$$

where $a$ and $b$ are the major and minor axis and $c$ is the maximum depth of the semi-prolate. This shape is chosen to optimize the efforts for mesh generation without loosing the general three-dimensional characteristics of the model. To reduce the number of figures, only the results for cross sections $y=0$ and $x=0$ are presented, and they will be referred to as sections $\mathrm{A}$ and $\mathrm{B}$, respectively. Incident waves are plane $\mathrm{P}, \mathrm{SV}, \mathrm{SH}$, and Rayleigh waves. The azimuthal angle of incidence $\phi$ is measured counterclockwise from negative $x$ axis (Fig. 1(a)). The off-vertical angle of incidence $\theta$ is measured from positive $z$ axis toward vector $r$, which defines the azimuthal position on the surface of the half-space. The amplitude of the incident waves are the same as those used by Dravinski and Mossessian (1987a). For convenience, a dimensionless frequency of incident wave $\Omega_{\mathrm{a}}$ is defined as the ratio of the maximum length of the basin $2 a$ and the 
wavelength of shear waves in the half-space. Throughout the paper $\mu_{j}, \alpha_{j}, \beta_{j}$; $j=0,1, \cdots, R$ denote the shear modulus, $\mathrm{P}$ - and S-wave velocities, respectively for layer $D_{j}$ (see Fig. 1). Subscript zero refers to the half-space while nonzero ones denote the dipping layers.

Several parameters may greatly affect the numerical accuracy of the method. These are: i) shape and location of the auxiliary surface $S_{\mathrm{a}}$; ii) number of sources (point forces) $M$; iii) number of observation points $N$; and iv) maximum length of each element. From previous investigations (Dravinski and Mossessian, 1987 b; Mossessian and Dravinski, 1987) it has been determined that a good choice for the auxiliary surface $S_{\mathrm{a}}$ is the one that follows in shape the boundary B. Therefore, the auxiliary surface is chosen to be in the following form:

$$
\begin{aligned}
& x_{\mathrm{a}}=(1-\xi) x, \\
& y_{\mathrm{a}}=(1-\xi) y, \\
& z_{\mathrm{a}}=(1-\xi) z,
\end{aligned}
$$

where $x, y$, and $z$ are described by Eqs. (26), (27). Through numerical experiments the value of the parameter $\xi$ is chosen to be 0.4 . The number of sources is 105 and the number of observation points (same as the boundary nodes at $B$ ) is 295 . The effect of element size is discussed in later sections.

\subsection{Finite element discretization}

The finite element mesh form at elliptical cross section $z=0$ is shown in Fig. 2(a). Due to symmetry only a quarter of the mesh plane is displayed. This mesh form is
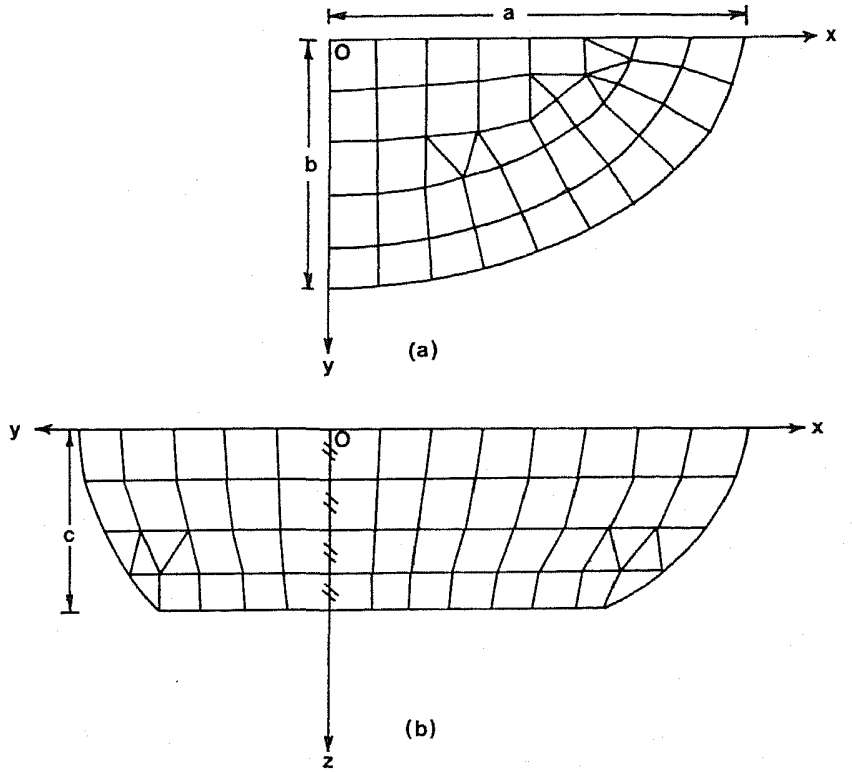

Fig. 2. Finite element meshes for sections $x-y, x-z$, and $y-z$ of the basin.

Vol. 40 , No. 1, 1992 
repeated for deeper sections following the pattern shown in Fig. 2(b), which displays the view of the mesh distributions along the vertical sections $x-z$ and $y-z$. Again, due to symmetry only half of each section is displayed. It is apparent from Fig. 2(a) and (b) that this model can easily incorporate planar and nonplanar layering. Throughout volume discretization only eight node cubic and six node triangular prisms are used. The number of elements used is 768 , which leads to a total of 835 nodes. The mesh size is kept constant for all the examples presented in this study.

\subsection{Testing of the method}

In order to assess the accuracy of the method the case of a homogeneous dipping layer subjected to various incident wave fields is considered first. For this problem the results of the hybrid technique are compared to the ones obtained through an indirect boundary integral equation method (Mossessian and Dravinski, 1990). Surface response at section $\mathrm{A}$ for incident $\mathrm{P}, \mathrm{SV}, \mathrm{SH}$, and Rayleigh waves with azimuthal angle of incidence $\phi=0^{\circ}$ is shown in Fig. 3. It can be seen from these figures that for all incident waves the results of hybrid technique are in excellent agreement with those obtained by the boundary integral equation method. Keeping the element sizes constant and increasing the frequency by $30 \%$, results in displacement fields depicted by Fig. 4 . Some differences between the results of the two methods are apparent. These discrepancies are due to the fact that the number of elements per wavelength in the hybrid technique has decreased. For this model the finite element discretization error has been analyzed by considering the case when the material properties of the half-space are the same as the ones for the dipping layer. In this case no scattering takes place, hence the internal nodal displacements are obtained through Eq. (11) by using free-field displacement values for boundary nodes. Therefore, deviation of the internal nodal displacement values from the free-field ones would correspond entirely to the error generated by the finite element discretization. Using this approach, by keeping the mesh size constant and varying the frequency, it is established that 10 elements per wavelength are sufficient for convergence of the results.

In order to show the capability and versatility of the method, numerical results for inhomogeneous basins are presented next.

\subsection{Horizontally stratified basin}

A basin consisting of two homogeneous strata is considered next (see Fig. 5(a)). This model is obtained from the homogeneous dipping layer considered in Figs. 3 and 4 by changing the material properties of the lower section of the basin to fall in between the ones of the top layer and the half-space. The surface displacement at section A for incident $\mathrm{P}, \mathrm{SV}, \mathrm{SH}$, and Rayleigh waves with azimuthal angle of incidence $\phi=0^{\circ}$ are depicted by Fig. 6. Comparison of the results with the ones for homogeneous basin (Fig. 4) reveals that introduction of the intermediate layer has considerably reduced the amplification at the center of the valley. This is not surprising since the "average velocity of the basin" (Bard and Gariel, 1986) has become larger than the case of the homogeneous dipping layer. It is interesting to note that for the Rayleigh waves the horizontal component of displacement has been affected very little by stratification while the changes for vertical component of motion are drastic. For different incident 

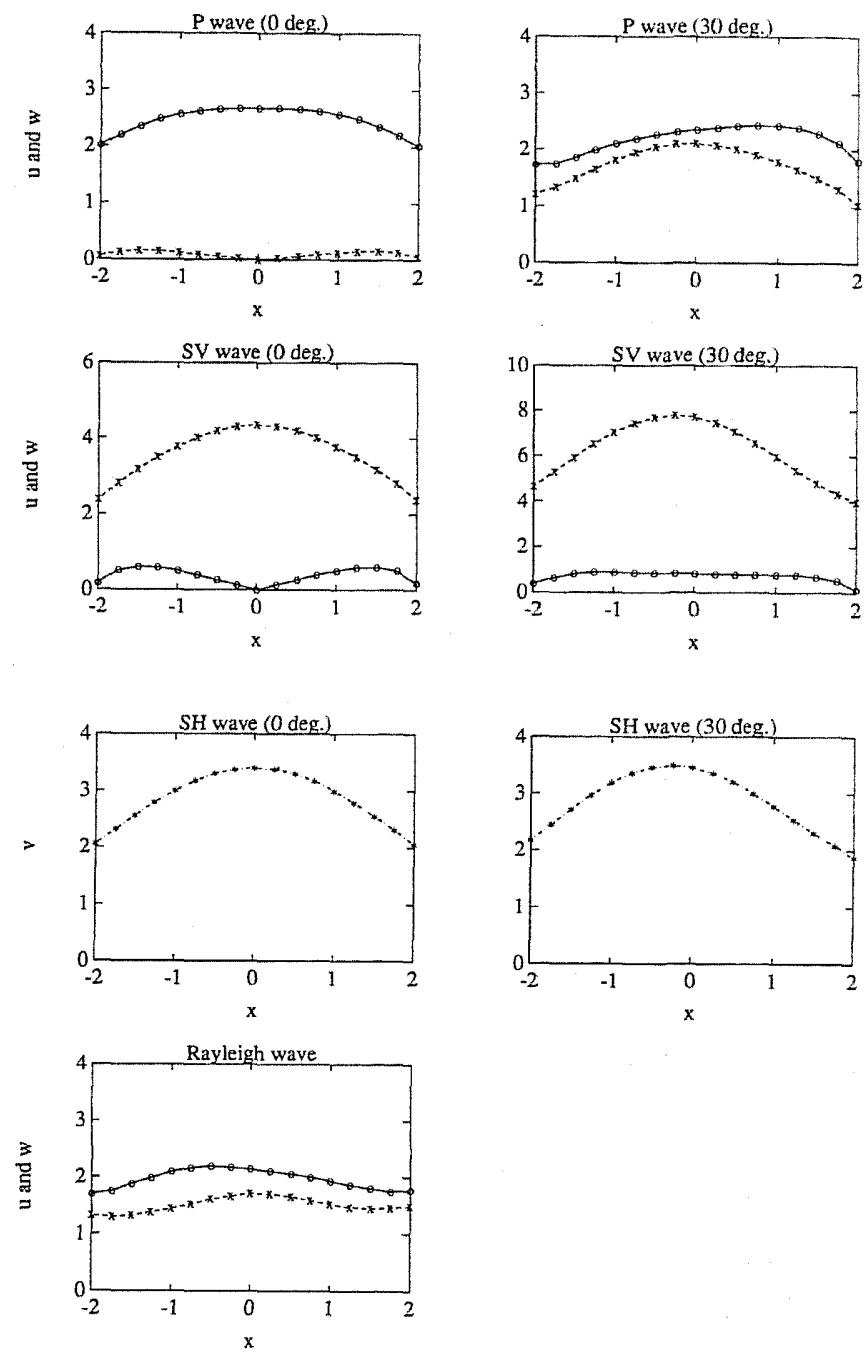

Fig. 3. Amplitudes of surface displacement spectra for a homogeneous valley at section $\mathrm{A}$ for incident $\mathrm{P}, \mathrm{SV}, \mathrm{SH}$, and Rayleigh waves with $\phi=0^{\circ}, \theta=0^{\circ}, 30^{\circ}$. $a=2, b=1.2, c=0.88, \mu_{0}=\beta_{0}=1, \alpha_{0}=2$, and $\mu_{1}=1 / 6, \beta_{1}=0.5, \alpha_{1}=1, \Omega_{\mathrm{a}}=0.64$ (Unless stated differently these parameters stay the same for other figures). Dash, dash-dot, and solid lines represent $x$-, $y$-, and $z$-components $(u, v, w)$ of the displacement vector, respectively, obtained by the hybrid technique (This convention holds for all the figures). The ' $x$ ' signs, stars, and open circles denote $x-, y$-, and $z$-components of the displacement vector, respectively, obtained by the boundary integral equation method.

Vol. 40 , No. 1, 1992 

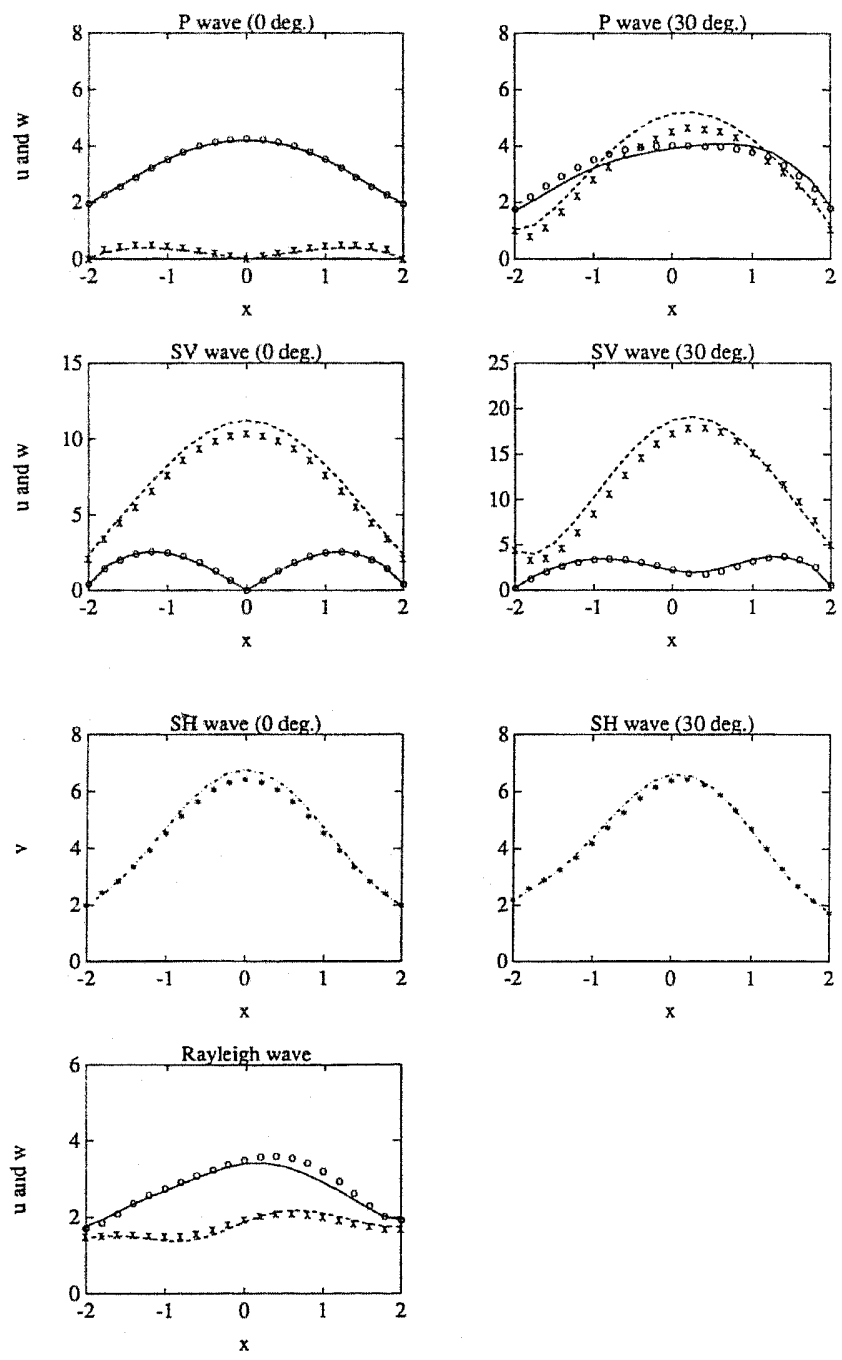

Fig. 4. Amplitudes of surface displacement spectra for a homogeneous valley at section A for incident P, SV, SH, and Rayleigh waves. $\phi=0^{\circ}, \Omega_{\mathrm{a}}=0.83$.

waves the surface responses at section B are shown by Fig. 7. Coupling between P/SV and SH modes appear to be much stronger for incident $\mathrm{SH}$ wave than the other type of waves.

\subsection{Multiple dipping layer basin}

To further emphasize the versatility of the method, a case of a basin with two dipping layers is considered. This model is obtained from the horizontally layered basin studied earlier. Namely, the lower layer is extended toward the surface of the half-space 

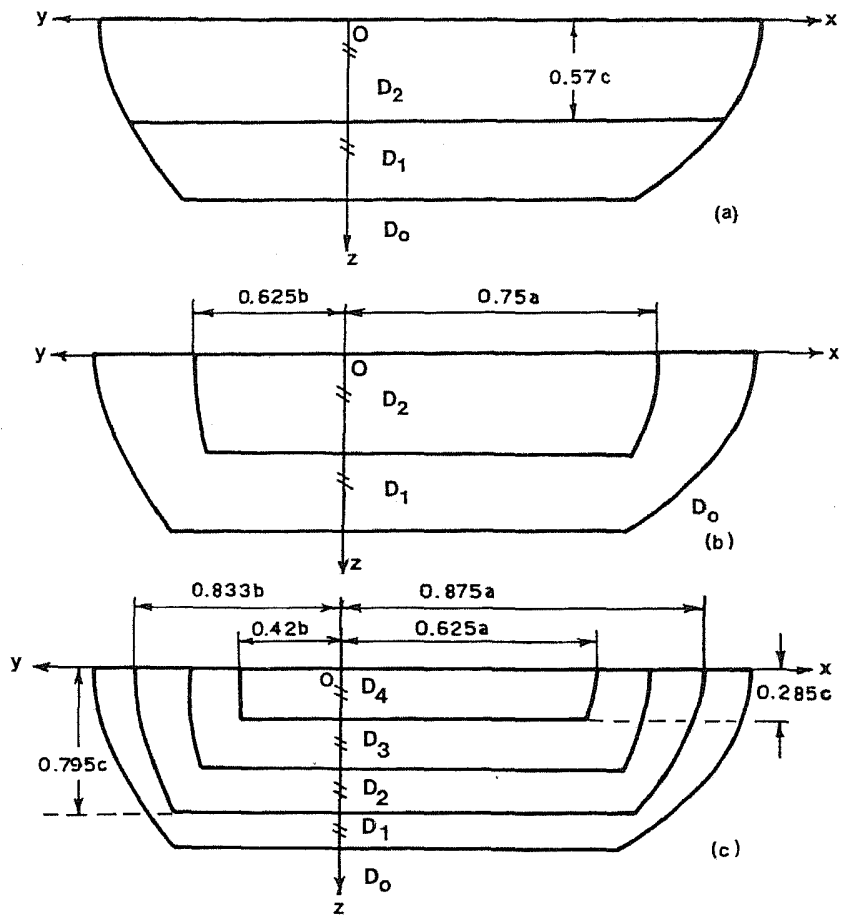

Fig. 5. Three models of inhomogeneous basins.

through a circumferential strip (see Fig. 5(b)). Surface displacements at section A for various incident waves with azimuthal angle of incidence $\phi=0^{\circ}$ are displayed by Fig. 8. In comparison with the results of the horizontally stratified basin, here the overall amplifications are smaller. It should be noted that the observed changes in the slopes are due to discontinuity of the material properties at the interface of the two layers. Figure 9 shows the surface response at section B for various incident waves. Here again the amplification of the predominant component of motion is lower than the corresponding case of horizontally stratified basin (Fig. 7), and it is more confined to surface of the top layer. However, the intensity of P/SV-SH mode conversion is about the same. For a four dipping layer basin, Fig. 10 shows the surface response at section A due to various incident waves. This model is obtained through simple extension of the two-layer model. Namely, an intermediate layer is added to each of the two layers (see Fig. 5(c)). In comparison with the case of two dipping layers, here the amplification of predominant motion is higher. However, the changes are rather small. This is partly due to the fact that the average velocity of the four dipping layers is close to that of the two dipping layers and also due to the fact the frequency of incident wave is rather low.

The complexity of the problems discussed here clearly demonstrate the capability of the hybrid technique. Furthermore, it is of interest to note that the computational time needed for the hybrid technique in the case of two and four dipping layers (Figs. 

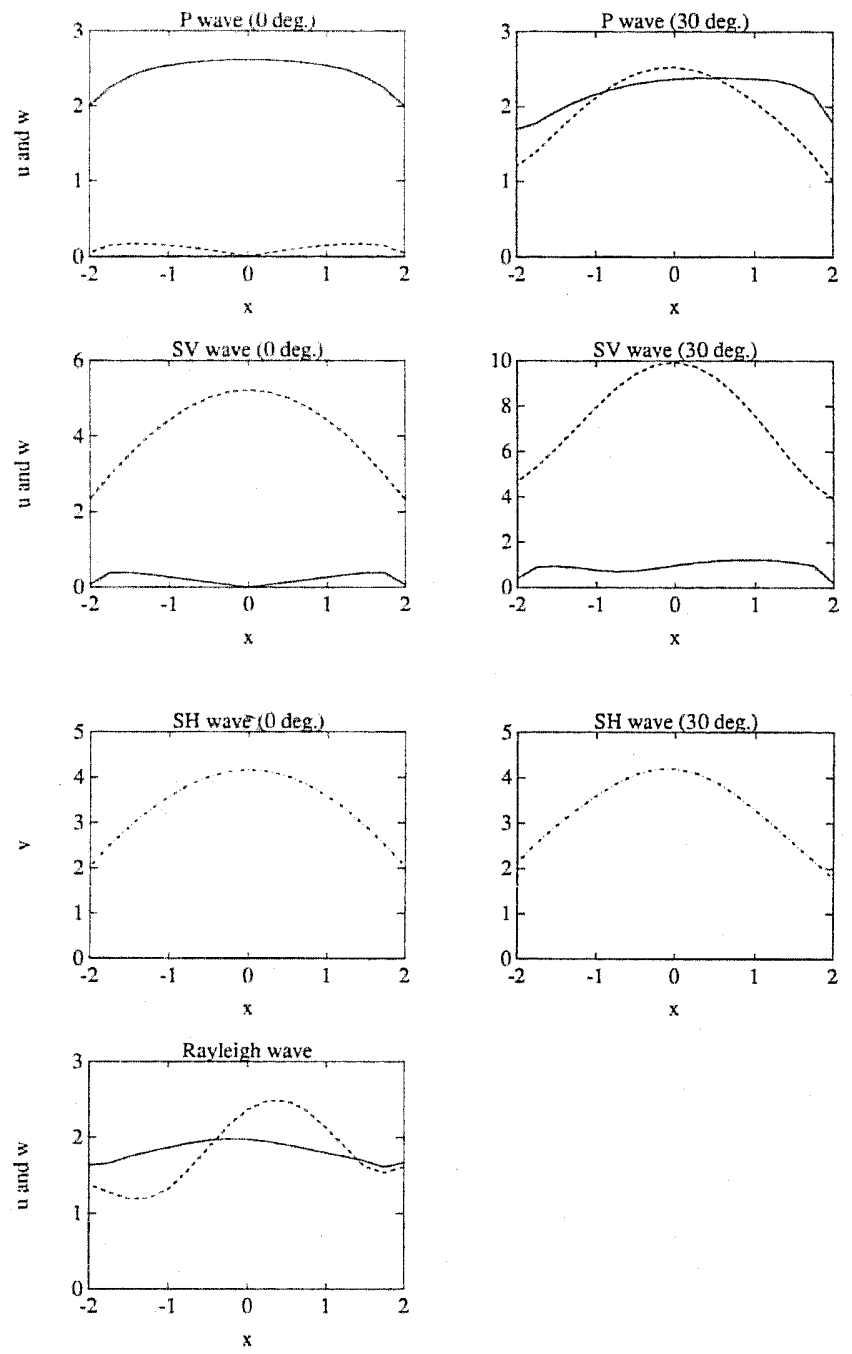

Fig. 6. Amplitudes of surface displacement spectra at section $\mathrm{A}$ of a basin with two horizontal layers and incident P.SV, SH, and Rayleigh waves. $\mu_{0}=\beta_{0}=1$, $x_{0}=2, \mu_{1}=0.45, \beta_{1}=0.73, \alpha_{1}=1.46, \mu_{2}=1 / 6, \beta_{2}=0.5, \alpha_{2}=1, \Omega_{\mathrm{a}}=0.83, \phi=0^{\circ}$.

8,9 , and 10) is the same as in the case of homogeneous basin (one dipping layer, Fig. 4). However, for the boundary integral equation method, in going from one to two and four dipping layers (in the analogous model), the required computational time would approximately increase by factors of 8 and 64, respectively. This is caused by increase in size of the Green's functions matrix. This analogy is true for other boundary methods such as wave function expansion approach (Eshraghi and Dravinski, 1988 a, b). Such comparisons illustrate that the hybrid technique tends to be more efficient than the boundary integral equation approach as the structure of the inhomogeneity becomes 

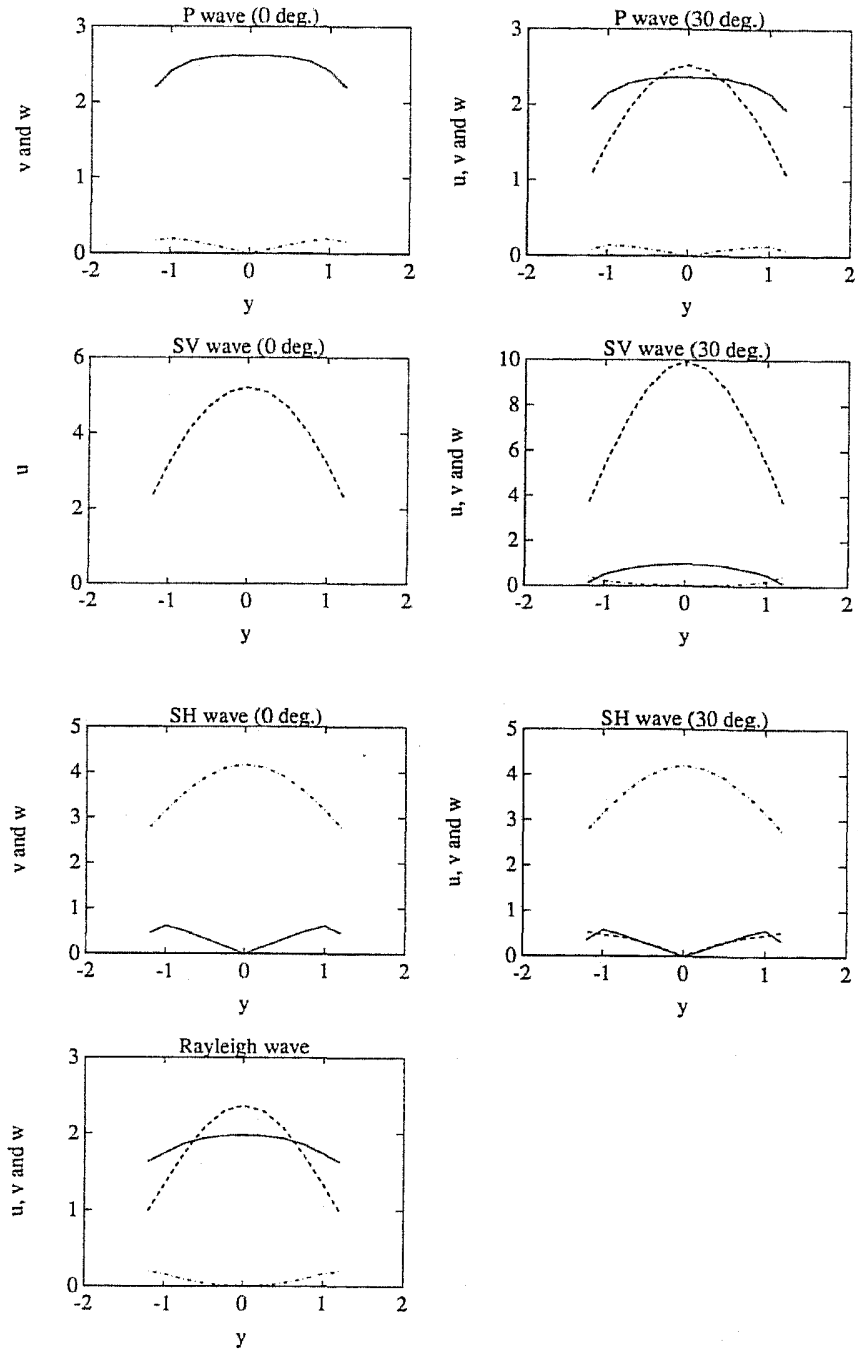

Fig. 7. Amplitudes of surface displacement spectra at section B of the basin with two horizontal layers and incident $\mathrm{P}, \mathrm{SV}, \mathrm{SH}$, and Rayleigh waves. $\Omega_{\mathrm{a}}=0.83$, $\phi=0^{\circ}$.

more complex. The drawback of the hybrid method is that the required number of nodes grows rapidly as the frequency increases. Therefore it appears that for simple inhomogeneities it may be more beneficial to use boundary integral equation approach (specially for higher frequencies). However, if the inhomogeneity is very complex (e.g., a basin with a number of dipping layers, heterogeneity, or anisotropicity) the hybrid technique becomes much more effective.

Vol. 40, No. 1, 1992 

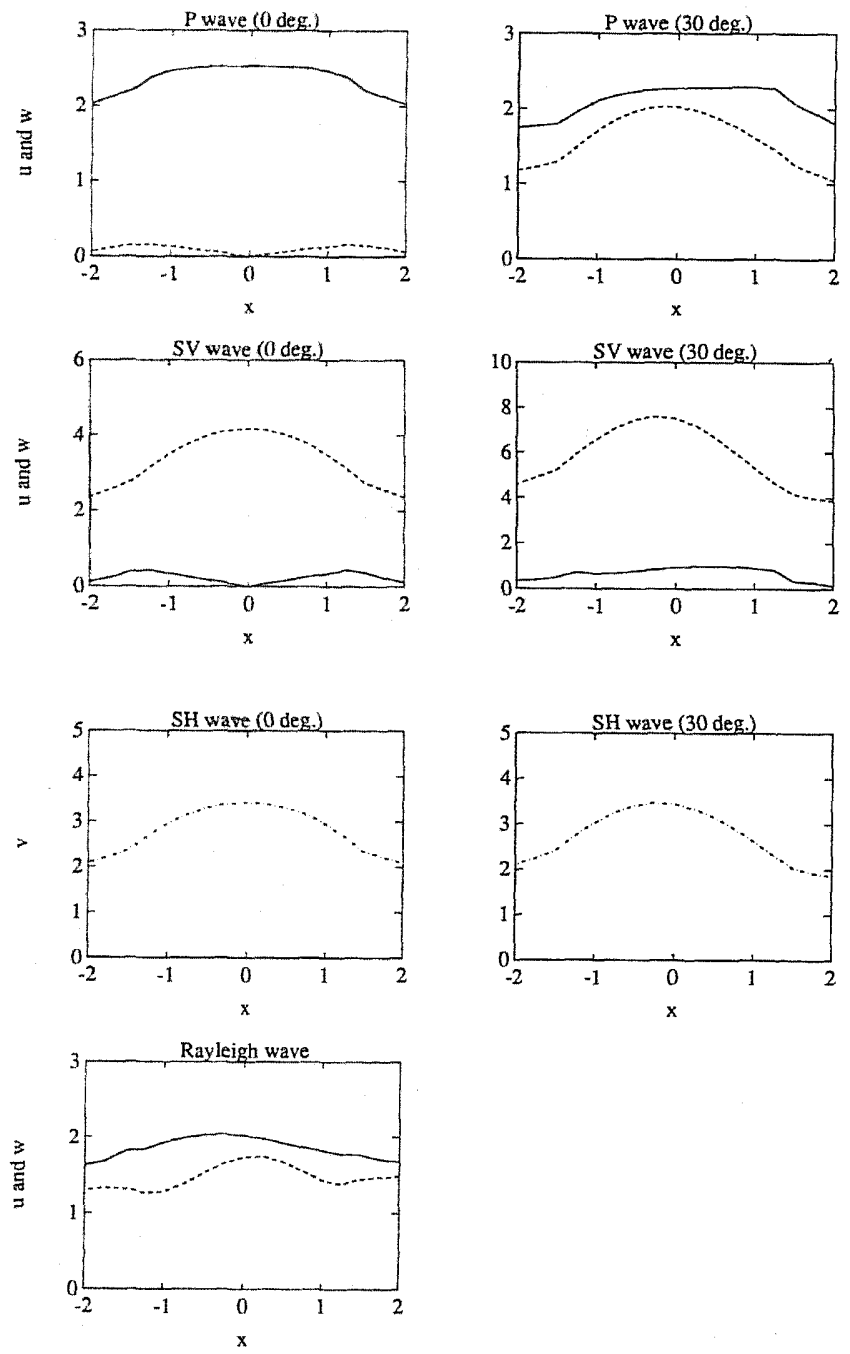

Fig. 8. Amplitudes of surface displacement spectra at section $\mathrm{A}$ of the basin with two dipping layers and incident $\mathrm{P}, \mathrm{SV}, \mathrm{SH}$, and Rayleigh waves. $\Omega_{\mathrm{a}}=0.83$, $\phi=0^{\circ}$.

\section{Summary and Conclusion}

A hybrid method which combines an indirect boundary integral equation and the finite element techniques, has been developed to study scattering of elastic waves by general three-dimensional subsurface inhomogeneities. The application of the method are demonstrated through studying the surface responses of elastic basins containing several horizontal or dipping layers subjected to $\mathrm{P}, \mathrm{SV}, \mathrm{SH}$, and Rayleigh waves.

Comparisons of the results obtained by the present method with those obtained by 

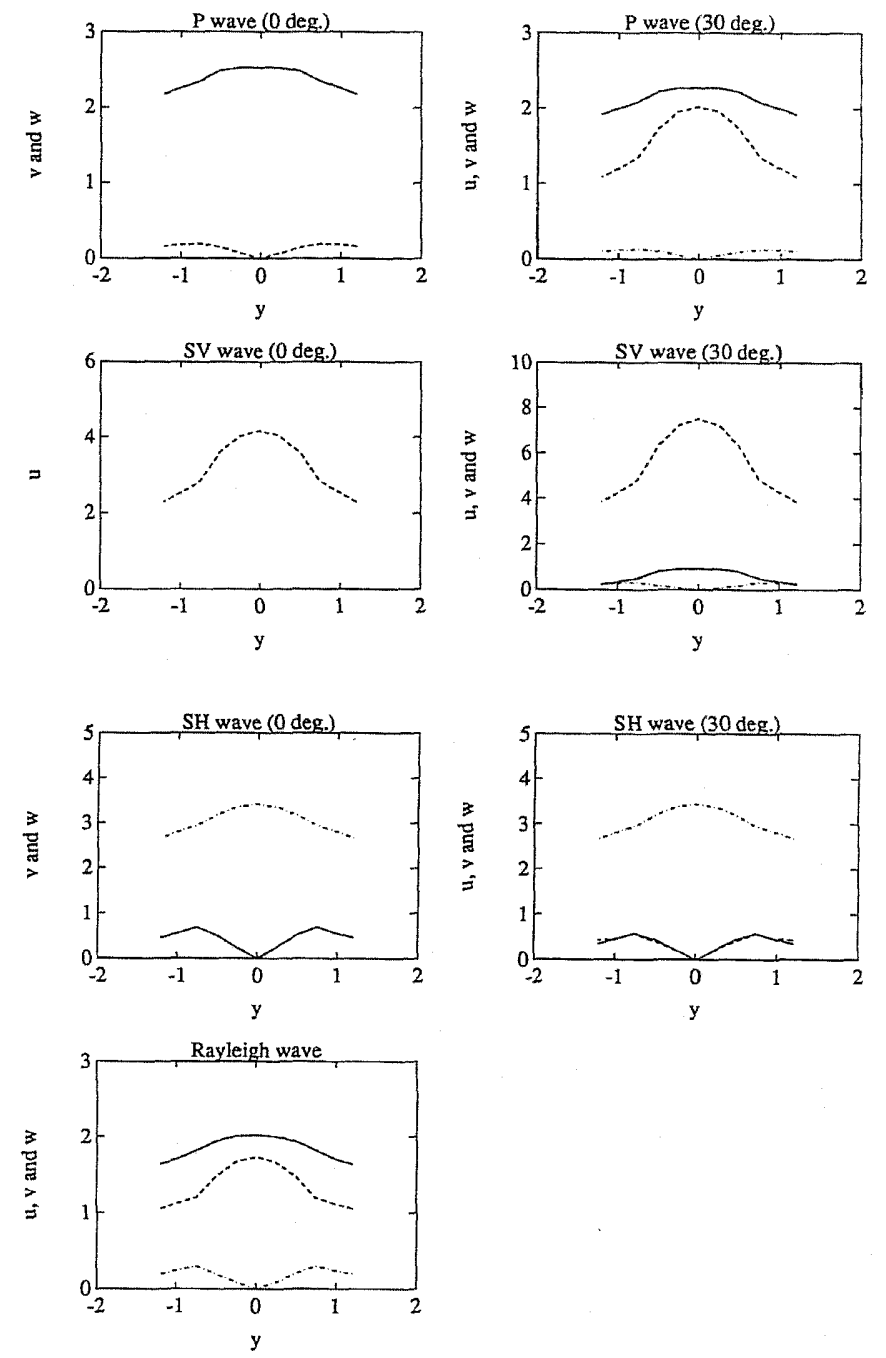

Fig. 9. Amplitudes of surface displacement spectra at section B of the basin with two dipping layers and incident $\mathrm{P}, \mathrm{SV}, \mathrm{SH}$, and Rayleigh waves. $\Omega_{\mathrm{a}}=0.83$, $\phi=0^{\circ}$.

a boundary integral equation method, clearly show that the proposed technique produces very accurate results, provided that the condition of minimum number of elements per wavelength is satisfied (about 10 elements per wavelength). The types of problems considered here indicate that the method can easily handle dipping layers with very irregular geometries or varying material properties. Furthermore, it is demonstrated that the hybrid technique becomes much more efficient than the boundary integral equation method as the structure of the inhomogeneities becomes very complex. The drawback of the hybrid method is that the memory requirement increases very rapidly

Vol. 40, No. 1, 1992 

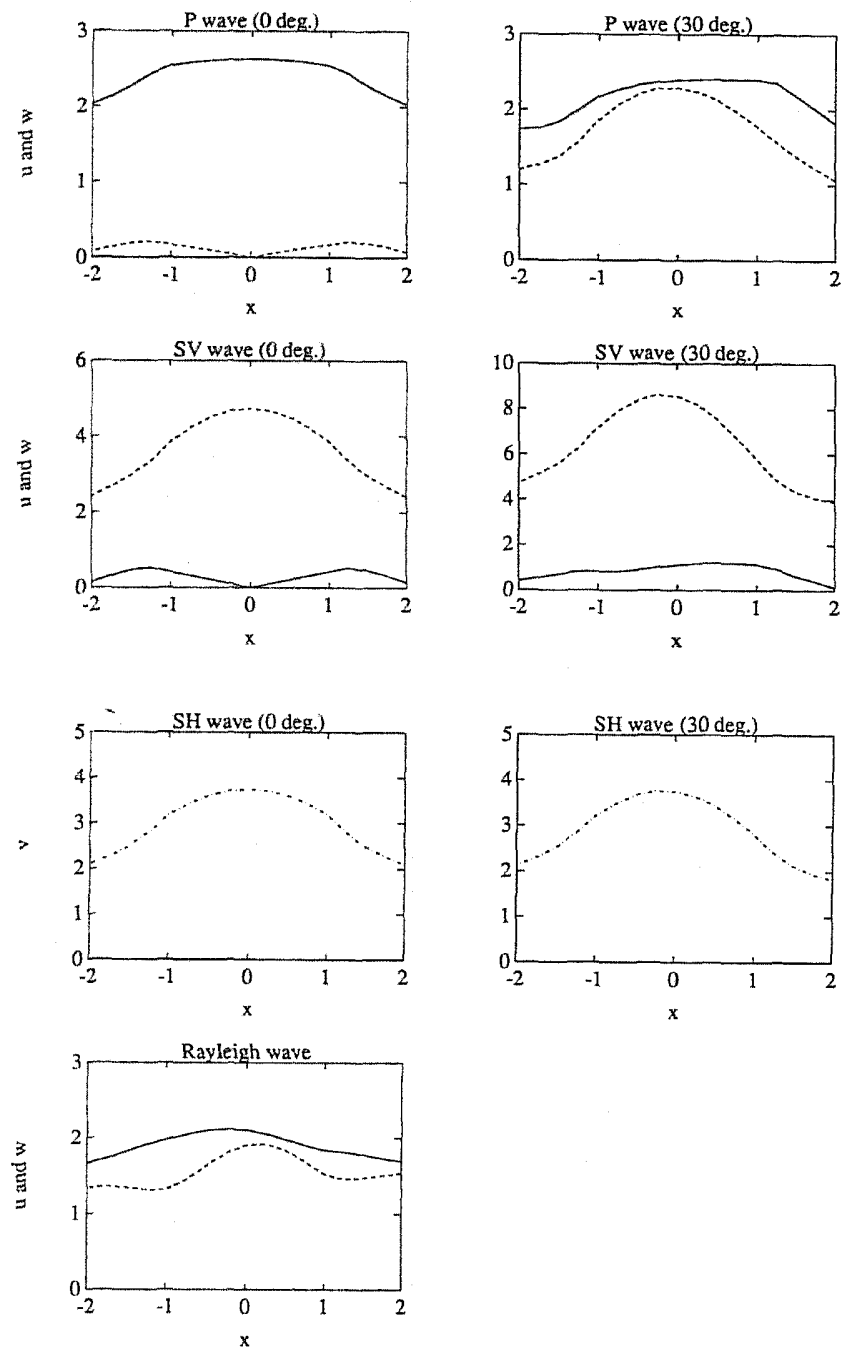

Fig. 10. Amplitudes of surface displacement spectra at section $\mathrm{A}$ of the basin with four dipping layers and incident $\mathrm{P}, \mathrm{SV}, \mathrm{SH}$, and Rayleigh waves. $\Omega_{\mathrm{a}}=0.83$, $\phi=0^{\circ}, \mu_{0}=\beta_{0}=1, \alpha_{0}=2, \mu_{1}=0.392, \beta_{1}=0.7, \alpha_{1}=1.4, \mu_{2}=0.284, \beta_{2}=0.62$, $\alpha_{2}=1.24, \mu_{3}=0.198, \beta_{3}=0.54, \alpha_{3}=1.08$, and $\mu_{4}=0.131, \beta_{4}=0.46, \alpha_{4}=0.92$.

(for the finite element part) as the frequency increases. However, with the existing rapid growth in computational facilities this obstacle is expected to be remedied to a great extent in the near future.

This work was supported in part by the National Science Foundation under Grant No. 53-4519-3792 and in part by the Office of Naval Research under Contract No. N00014-88-K-0157. 


\section{REFERENCES}

Aki, K., Local site effects on strong ground motion, Proceedings of Earthquake Engineering and Soil Dynamics II, American Society Civil Engineering., Park City, Utah, June 27-30, 103-155, 1988.

Aki, K. and K. L. Larner, Surface motion of a layered medium having an irregular interface due to incident plane SH waves, J. Geophys. Res., 75, 933-954, 1970.

Aki, K. and P. G. Richards, Quantitative Seismology, Vol. 1, W. H. Freeman \& Co., San Francisco, California, 1980.

Anderson, J. G., P. Bodin, J. N. Brune, J. Prince, S. K. Singh, R. Quaas, and M. Onate, Strong ground motion from the Michoacan, Mexico, earthquake, Science, 233, 1043-1049, 1986.

Bard, P.-Y. and M. Bouchon, The seismic response of sediment-filled valleys. Part I. The case of incident SH waves, Bull. Seismol. Soc. Am., 70, 1263-1286, 1980 a.

Bard, P.-Y. and M. Bouchon, The seismic response of sediment-filled valleys. Part II. The case of incident P and SV waves, Bull. Seismol. Soc. Am., 70, 1921-1941, 1980 b.

Bard, P.-Y. and M. Bouchon, The two dimensional resonance of sediment-filled valleys, Bull. Seismol. Soc. Am., 75, 519-541, 1985.

Bard, P.-Y. and J.-C. Gariel, The seismic response of two-dimensional sedimentary deposits with large vertical velocity gradients, Bull. Seismol. Soc. Am., 76, 343-346, 1986.

Berg, A. P., A hybrid solution for wave propagation problems in regular media with bounded irregular inclusions, Geophys. J. R. Astron. Soc., 79, 3-10, 1984.

Beskos, D. E., Boundary element method in dynamic analysis, Appl. Mech. Rev., 40, 1-23, 1987.

Beskos, D. E. and C. C. Spyrakos, Dynamic response of strip foundations by the time domain BEM-FEM method, Final Report Part B, NSF Grant No. CEE-8024725, Department of Civil and Mineral Engineering, University of Minnesota, Minneapolis MN, Jan., 1984.

Bouchon, M., Effect of topography on surface motion, Bull. Seismol. Soc. Am., 63, 615-632, 1973.

Bouchon, M. and K. Aki, Near-field of a seismic source in a layered medium with irregular interfaces, Geophys. J. R. Astron. Soc., 50, 669-684, 1977.

Cole, D. M., D. D. Kosloff, and J. B. Minster, A numerical boundary integral equation method for elastodynamics, Bull. Seismol. Soc. Am., 68, 1331-1357, 1978.

Dravinski, M., Influence of interface depth upon strong ground motion, Bull. Seismol. Soc. Am., 72, 596-614, 1982 a.

Dravinski, M., Scattering of SH waves by subsurface topography, J. Eng. Mech. Div., 108, No. EM1, 1-16, 1982 b.

Dravinski, M., Scattering of elastic waves by an alluvial valley, J. Eng. Mech. Div., 108, No. EM1, 19-31, $1982 \mathrm{c}$.

Dravinski, M., Scattering of plane harmonic SH waves by dipping layers of arbitrary shape, Bull. Seismol. Soc. Am., 73, 1303-1319, 1983.

Dravinski, M. and T. K. Mossessian, Scattering of harmonic P, SV, and Rayleigh waves by dipping layers of arbitrary shape, Bull. Seismol. Soc. Am., 77, 212-235, 1987 a.

Dravinski, M. and T. K. Mossessian, Amplification of surface ground motion by an inclusion of arbitrary shape for harmonic surface line loading, Numer. Methods Part. Diff. Eqs., 3, 9-27, $1987 \mathrm{~b}$.

Dravinski, M. and T. K. Mossessian, On evaluation of the Green functions for harmonic line loads in a viscoelastic half space, Int. J. Numer. Methods Eng., 26, 823-841, 1988.

Eshraghi, H. and M. Dravinski, Transient scattering of elastic waves by dipping layers of arbitrary

Vol. 40, No. 1, 1992 
shape. Part 1. Antiplane strain model, Earthq. Eng. Struct. Dyn., 18, 397-415, 1988 a.

Eshraghi, H. and M. Dravinski, Transient scattering of elastic waves by dipping layers of arbitrary shape. Part 2. Plane strain model, Earthq. Eng. Struct. Dyn., 18, 417-434, $1988 \mathrm{~b}$.

Herrera, I. and F. J. Sabina, Connectivity as an alternative to boundary integral equations: Construction of basis, Proc. Natl. Acad. Sci. U.S.A., 75, 2059-2063, 1978.

Hong, T. L. and D. V. Helmberger, Glorified optics and wave propagation in nonplanar structure, Bull. Seismol. Soc. Am., 68, 1313-1330, 1978.

Kagami, H., C. M. Duke, G. C. Liang, and Y. Ohta, Observation of 1- to 5-second microtremors and their application to earthquake engineering. Part II. Evaluation of site effect upon seismic wave amplification due to extremely deep soil deposits, Bull. Seismol. Soc. Am., 72, 987-998, 1982.

Kagami, H., S. Okada, K. Shiono, M. Oner, M. Dravinski, and A. Mal, Observation of 1- to 5-second microtremors and their application to earthquake engineering. Part III. A two dimensional study of site effect in the San Fernando Valley, Bull. Seismol. Soc. Am., 76, 1801-1812, 1986.

Kawase, $\mathrm{H}$. and $\mathrm{K}$. Aki, A study on the response of a soft basin for incident $\mathrm{S}, \mathrm{P}$, and Rayleigh waves with special reference to the long duration observed in Mexico City, Bull. Seismol. Soc. Am., 79, 1361-1382, 1989.

King, J. L. and B. E. Tucker, Observed variations of earthquake motion across a sediment-filled valley, Bull. Seismol. Soc. Am., 74, 137-151, 1984.

Kobayashi, S., N. Nishimura, and K. Mori, Applications of boundary element-finite element combined method to three dimensional viscoelastodynamic problems, in Boundary Elements, ed. D. Qinghua, pp. 67-74, Pergamon Press, Oxford, 1986.

Moczo, P., P.-Y. Bard, and I. Pšenčík, Seismic response of a two dimensional structure by the ray method, J. Geophys., 62, 38-49, 1987.

Mossessian, T. K., Diffraction of elastic waves by non-axisymmetric three dimensional subsurface inhomogeneities using a boundary integral equation method and a hybrid technique, $\mathrm{Ph} . \mathrm{D}$. thesis, Department of Mechanical Engineering, University of Southern California, Los Angeles, 1989.

Mossessian, T. K. and M. Dravinski, Application of a hybrid method for scattering of P, SV and Rayleigh waves by near surface irregularities, Bull. Seismol. Soc. Am., 77, 1784-1803, 1987.

Mossessian, T. K. and M. Dravinski, Scattering of elastic waves by three dimensional surface topographies, Wave Motion, 11, 579-592, 1989.

Mossessian, T. K. and M. Dravinski, Amplification of elastic waves by a three dimensional valley. Part 1: Steady-state response, Earthq. Eng. Struct. Dyn., 19, 667-680, 1990.

Niwa, Y. and S. Hirose, Application of the BEM to elastodynamics in a three dimensional half space, Recent Applications in Computational Mechanics, ASCE, 1-15, 1987.

Niwa, Y., S. Hirose, and M. Kitahara, Application of the boundary integral equation (BIE) method to transient analysis of inclusions in a half-space, Wave Motion, 8, 77-91, 1986.

Nowack, R. and K. Aki, The two dimensional Gaussian beam synthetic method: Testing and application, J. Geophys. Res., 89, 7797-7819, 1984.

Repetto, P., I. Arango, and H. B. Seed, Influence of site characteristics on building damage during the October 3, 1974, Lima earthquake, Report No. UCB/EERC 80/41, University of California, Berkeley, California, 1980.

Rizzo, F. J., D. J. Shippy, and M. Rezayat, A boundary integral equation method for radiation 
and scattering of elastic waves in three dimensions, Int. J. Numer. Methods Eng., 21, 115-129, 1985.

Sánchez-Sesma, F. J., Diffraction of elastic waves by three-dimensional surface irregularities, Bull. Seismol. Soc. Am., 73, 1621-1636, 1983.

Sánchez-Sesma, F. J. and E. Rosenblueth, Ground motion at canyons of arbitrary shapes under incident SH-waves, Earthq. Eng. Struct. Dyn., 7, 441-449, 1979.

Smith, W. D., The application of finite element analysis to body wave propagation problems, Geophys. J. R. Astron. Soc., 42, 747-768, 1975.

Trifunac, M. D., Surface motion of semicylindrical alluvial valley for incident plane SH waves, Bull. Seismol. Soc. Am., 61, 1755-1770, 1971.

Wong, H. L., Diffraction of P, SV, and Rayleigh waves by surface topographies, Bull. Seismol. Soc. Am., 72, 1167-1184, 1982.

Wong, K. C., A. H. Shah, and S. K. Datta, Diffraction of elastic waves in a half space. II. Analytical and numerical solutions, Bull. Seismol. Soc. Am., 75, 69-91, 1985.

Zahradnik, J. and F. Hron, Seismic ground motion of sedimentary valleys-example La Molina, Lima, Peru, J. Geophys., 62, 31-37, 1987.

Zeevaert, L., Strong ground motion recorded during earthquakes of May the 11th and 19th, 1962 in Mexico City, Bull. Seismol. Soc. Am., 54, 209-231, 1964.

Zienkiewicz, O. C., The Finite Element Method (3rded.), McGraw Hill Book Company, London, 1977.

Zienkiewicz, O. C., D. W. Kelly, and P. Bettess, The coupling of the finite element method and boundary solution procedures, Int. J. Numer. Methods Eng., 11, 355-375, 1977.

Vol. 40, No. 1, 1992 